\title{
INÉGALITÉ DE MARKOV EN PLUSIEURS VARIABLES
}

\author{
WIESŁAW PLEŚNIAK
}

Reçu le 16 mai 2005; Révisé le 13 mars 2006; Accepté le 26 avril 2006

L'inégalité de Markov joue un rôle important en théorie constructive de fonctions. Dans les deux dernières décennies, on a développé sa théorie multidimensionnelle. Le but de cet article est de présenter le récent progrès de cette belle théorie.

Copyright (c) 2006 Hindawi Publishing Corporation. All rights reserved.

\section{Inégalité de Markov}

1.1. Introduction. En 1889, Andrei Andreievitch Markov a répondu à une question posée deux ans plus tôt par Mendeleev en montrant que, pour tout polynôme $p \in \mathbb{C}[x]$, on a

$$
\left|p^{\prime}(x)\right| \leq(\operatorname{deg} p)^{2}\|p\|_{[-1,1]} \quad \text { pour } x \in[-1,1]
$$

où $\|p\|_{I}=\sup |p|(I)$. Ce résultat est optimal puisque, pour les polynômes de Tchebyschev

$$
T_{k}(x)=\cos k \arccos x \quad(x \in[-1,1]), k=1,2, \ldots,
$$

on a $\left\|T_{k}\right\|_{[-1,1]}=1$ et $\left|T_{n}^{\prime}( \pm 1)\right|=n^{2}$. L'inégalité de Markov a été le point de départ d'une énorme littérature. En effet, elle a de nombreuses applications en analyse et en physique. Pour des détails on peut se reporter à l'ouvrage de Rahman et Schmeisser ([44]).

La théorie correspondante en plusieurs variables est relativement nouvelle : avant 1980, toutes les extensions connues de l'inégalité de Markov, dans la pratique, concernaient uniquement des ensembles compacts, convexes et d'intérieur non vide de $\mathbb{R}^{n}$ (voir, e.g., Baouendi-Goulaouic [2]). Le principal obstacle à l'étude des cas plus généraux provenait de l'existence, dans $\mathbb{R}^{n}$, d'ensembles à pointes n'admettant aucune extension multidimensionnelle de l'inégalité de Markov. Cette remarque est due à Zerner ([55]).

Exemple 1.1. On pose $E=\left\{(x, y) \in \mathbb{R}^{2}: 0<y \leq \exp (-1 / x), 0<x \leq 1\right\} \cup\{(0,0)\}$ et $P_{k}(x, y)=y(1-x)^{k}$ pour $k=1,2, \ldots$ On a donc $\operatorname{deg} P_{k}=k+1,\left\|\partial P_{k} / \partial y\right\|_{E}=1$, tandis 
que $\left\|P_{k}\right\|_{E}<\exp (-\sqrt{k})$ pour $k=1,2, \ldots$. Par conséquent, il n'existe pas de constantes $M>0$ et $r>0$ telles qu'on ait, pour tout $k$,

$$
\left\|\partial P_{k} / \partial y\right\|_{E} \leq M(k+1)^{r}\left\|P_{k}\right\|_{E}
$$

Il est alors naturel de chercher les parties de $\mathbb{R}^{n}$ pour lequelles il est possible d'établir des majorations de l'exemple 1.1. On est ainsi ramené à la définition suivante.

Définition 1.2. On dira qu'une partie compacte de $\mathbb{R}^{n}$ admet (ou presérve) l'inégalité de Markov, ou bien qu'elle est markovienne, s'il existe des constantes $M>0$ et $r>0$ telles que, pour tout polynôme $P \in \mathbb{R}\left[x_{1}, \ldots, x_{n}\right]$, on ait

$$
\|\operatorname{grad} P\|_{E} \leq M(\operatorname{deg} P)^{r}\|P\|_{E} .
$$

Une théorie satisfaisante de l'inégalité de Markov en plusieurs variables, abordant en particulier le cas d'ensembles à pointes, a été développée dans les dernières deux décennies par Pawłucki, Pleśniak, Goetgheluck, Baran, Białas-Cież, Jonsson, Bos, Levenberg, Milman, Taylor, Goncharov, Totik et autres. Donnons ici un exemple d'un compact markovien de $\mathbb{R}^{n}$ à pointes, dû à Goetgheluck ([24]).

Théorème 1.3. Soit, pour $k \geq 1$,

$$
E_{k}=\left\{(x, y) \in \mathbb{R}^{2}: 0 \leq y \leq x^{k}, 0 \leq x \leq 1\right\} .
$$

Alors l'ensemble $E_{k}$ est markovien, pour l'exposant $r=2 k$. De plus, la valeur $2 k$ est optimale.

L'ensemble $E_{k}$, défini ci-dessus, est un sous-ensemble semi-analytique de $\mathbb{R}^{2}$. On peut alors se demander si toute partie semi-analytique (plus général, sous-analytique) de $\mathbb{R}^{n}$ admet une inégalité de Markov. La réponse à cette question est fournie par la théorie de Pawłucki-Pleśniak présentée ci-dessous, dont le point de départ était l'exemple de Goetgheluck.

\subsection{Ensembles semi-analytiques, sous-analytiques et à pointes polynomiales}

Définition 1.4. Une partie $E$ de $\mathbb{R}^{n}$ est dite semi-analytique si, pour tout $x \in \mathbb{R}^{n}$, on peut trouver un voisinage $U$ de $x$ et un nombre fini de fonctions réel analytiques $f_{i j}$ et $g_{i j}$ définies dans $U$, telles que

$$
E \cap U=\bigcup_{i} \bigcap_{j}\left\{f_{i j}>0, g_{i j}=0\right\}
$$

La projection d'un semi-analytique n'est pas nécessairement semi-analytique (Łojasiewicz ([34])). On appelle classe d'ensembles sous-analytiques, la famille obtenue en ajoutant à la famille des semi-analytiques, toutes les projections d'ensembles semianalytiques. Plus précisement,

Définition 1.5. Un sous-ensemble $E$ de $\mathbb{R}^{n}$ est dit sous-analytique si, pour tout $x \in \mathbb{R}^{n}$, il existe un voisinage $U$ de $x$ tel que $E \cap U$ soit la projection d'un semi-analytique borné $A$ de $\mathbb{R}^{n+m}$, où $m$ est un entier non négatif. 
L'ensemble $E_{k}$ de Goetgheluck est, de façon évidente, semi-analytique, et à fortiori sous-analytique, tandis que l'ensemble de Zerner est trop plat à l'origine pour être semianalytique. Il se trouve que les sous-analytiques n'admettent que des pointes polynomiales.

Définition 1.6. On dit qu'une partie $E$ de $\mathbb{R}^{n}$ est à pointes polynomiales (en bref, UPC= uniformly polynomially cuspidal) s'il existe des constantes positives $M$ et $m$, un entier positif $d$ et une application $h: \bar{E} \times[0,1] \rightarrow \bar{E}$, tels que, pour tout $x \in \bar{E}$, on ait $h(x, 1)=x$, la fonction $h(x, \cdot)$ soit un polynôme de degré $\leq d$ et

$$
\operatorname{dist}\left(h(x, t), \mathbb{R}^{n} \backslash E\right) \geq M(1-t)^{m} \quad \text { pour }(x, t) \in \bar{E} \times[0,1] .
$$

En utilisant le théorème de rectilinéarisation de Hironaka (voir [17]) et la séparation régulière de Łojasiewicz ([34]), on prouve (Pawłucki-Pleśniak [37])

Théorème 1.7. Tout ensemble sous-analytique borné de $\mathbb{R}^{n}$, à intérieur dense dans E, est UPC.

La famille des ensembles UPC est visiblement plus large que celle des sous-analytiques. Un exemple simple d'ensemble UPC qui n'est pas sous-analytique est fourni par $[0,1] \times$ $[-1,1] \backslash E$, où $E$ est l'ensemble de Zerner.

Les ensembles UPC admettent une fonction de Green pluricomplexe continue de façon hölderienne. Soit $E$ une partie compacte de $\mathbb{C}^{n}$, on pose, pour tout $z \in \mathbb{C}^{n}$,

$$
V_{E}(z)=\sup \left\{u(z): u \in \mathscr{L}\left(\mathbb{C}^{n}\right),\left.u\right|_{E} \leq 0\right\}
$$

où $\mathscr{L}\left(\mathbb{C}^{n}\right)=\left\{u \in \operatorname{PSH}\left(\mathbb{C}^{n}\right): \sup _{z \in \mathbb{C}^{n}}[u(z)-\log (1+|z|)]<\infty\right\}$ est la classe de Lelong des fonctions plurisous-harmoniques à croissance logarithmique. D'après la théorie du pluripotentiel due à Bedford-Taylor (voir [31]), si E est non pluripolaire (c'est-à-dire, si pour toute fonction $u \in \operatorname{PSH}\left(\mathbb{C}^{n}\right)$, l'inclusion $E \subset\{u(z)=-\infty\}$ implique $\left.u(z) \equiv-\infty\right)$, alors la régularisée supérieure

$$
V_{E}^{*}(z)=\limsup _{w \rightarrow z} V_{E}(w)
$$

de $V_{E}$ est un équivalent multidimensionnel de la fonction de Green classique pour $\mathbb{C} \backslash \widehat{E}$, où $\widehat{E}$ désigne l'enveloppe polynomiale de $E$.

L’importance de la classe UPC se traduit par le résultat suivant dû à Pawłucki-Pleśniak [37].

Théorème 1.8. Soit E une partie UPC compacte de $\mathbb{R}^{n}$, à paramètre $m$. On a

$$
V_{E}(z) \leq M(\operatorname{dist}(z, E))^{s}
$$

où $s=1 / 2[m]$ et $[m]:=k$ pour $k-1<m \leq k$ avec $k \in \mathbb{Z}$. 
Si $V_{E}$ est continue de façon HCP (abrév. de Hölder Continuity Property), en appliquant la formule intégrale de Cauchy, on montre que $E$ est markovien. Ceci entraîne (PawłuckiPleśniak [37])

Corollaire 1.9. Toute partie compacte $E$ de $\mathbb{R}^{n}$ de type UPC, en particulier tout sousanalytique compact $E \subset \mathbb{R}^{n}$, et d'intérieur dense dans $E$, est markovien.

Le résultat ci-dessus reste vrai dans le cadre, beaucoup plus général, des structures o-minimales polynomialement bornées. En particulier, si on considère une classe de fonctions quasi-analytiques de Carleman liée à une suite strictement logarithmiquement convexe, les ensembles définissables dans la structure de Rolin-Speissegger-Wilkie engendrée par cette classe sont UPC (voir Pierzchała [39]).

1.3. Dynamique complexe. La géométrie sous-analytique n'est pas seule à fournir des exemples d'ensembles markoviens. On peut en trouver plusieurs en dynamique complexe.

Soit $P: \mathbb{C}^{n} \rightarrow \mathbb{C}^{n}$ une application polynomiale, on définit l'ensemble de Julia rempli, associé à $P$, par

$$
J_{P}:=\left\{z \in \mathbb{C}^{n}:\left\{P^{j}(z)\right\}_{j \in \mathbb{N}_{0}} \text { est borné }\right\}
$$

Si l'exposant de Łojasiewicz de $P$ (à l'infini)

$$
\nmid(P):=\sup \left\{\delta: \liminf _{|z| \rightarrow \infty} \frac{|P(z)|}{|z|^{\delta}}>0\right\}
$$

est strictement plus grand que 1 , alors $J_{P}$ est HCP, d'où markovien (voir Kosek $[32,33]$ ).

Les ensembles markoviens décrits ci-dessus sont tous de type UPC, et donc HCP. Cependant, il y a des ensembles HCP qui ne sont pas UPC. Les premiers exemples de tels ensembles (de type Cantor) ont été construits par Jonsson ([29]). Il s'est avéré difficile de déterminer si le Cantor triadique classique est markovien ou non. Une réponse affirmative à ce problème a été fournie par Białas et Volberg ([13]) qui ont montré que cet ensemble est même de type HCP. Il convient de noter qu'il existe également des ensembles de type Cantor qui ne sont pas markoviens et pour lequels la fonction classique de Green est continue (voir Pleśniak [40], Goetgheluck-Pleśniak [26], Totik [52]). Quant à savoir si la propriété de Markov de $E$ entraîne la propriété HCP de $E$, ce problème reste toujours ouvert sauf pour des cas particuliers de $\mathbb{C}$ (voir Białas-Cież [14], Totik [52]). En général, on ne sait même pas si la propriété de Markov de $E$ entraîne la continuité de la fonction de Green $V_{E}$ ou la non-pluripolarité de E. Néanmoins, Białas-Cież ([15]) a montré que toute partie compacte de Markov de $\mathbb{C}$ est de capacité logarithmique positive, et par conséquent, qu'elle n'est pas polaire.

1.4. Relations avec les polynômes orthogonaux. On termine cette section par la mise en évidence des relations entre l'inégalité classique de Markov et les polynômes orthogonaux. Soit $E$ une partie compacte de $\mathbb{R}$ et soit $\mu$ une mesure de probabilité, à support $E$, telle que, pour tout disque $\Delta(x, r)$ centré en $x \in E$ de rayon $0<r \leq 1$, on ait 
$\mu(\Delta(x, r)) \geq c_{0} r^{s}$ avec des constantes $c_{0}>0$ et $s>0$ ne dépendant pas de $x$ et $r$. Soit $\left\{P_{n}: n=0,1,2, \ldots\right\}$ une suite de polynômes orthogonaux associée à $\mu$. On suppose que le degré de $P_{n}$ est $n$. On sait que, pour tout $n$, les zéros $\left\{x_{1}, x_{2}, \ldots, x_{n}\right\}$ du polynôme $P_{n}$ sont tous simples, réels et contenus dans le plus petit intervalle couvrant $E$. On a le résultat suivant dû à Jonsson (voir [30]).

Théorème 1.10. Le compact E est markovien si et seulement si l'on peut trouver des constantes $\gamma \geq 1, L>0$ et $\beta>0$ telles que

(i) il existe $d, 0<d \leq 1$, tel que, pour tout $x_{0} \in$ E et tout $r>0, r \leq 1$,

$$
\left\{x \in \mathbb{R}: d r^{\gamma} \leq\left|x-x_{0}\right| \leq r\right\} \cap E \neq \varnothing ;
$$

(ii) si $x_{i}$ et $x_{i-1}$ sont des zéros consécutifs de $P_{n}$, avec $n \geq 2$, alors

$$
\left|x_{i}-x_{i-1}\right| \geq L / n^{\beta}
$$

\section{Applications}

Nous allons présenter quelques applications importantes de l'inégalité de Markov. Nous commençons par

2.1. Approximation polynomiale de fonctions $\mathscr{C}^{\infty}$. Rappelons le théorème classique de Bernstein [12].

Théorème 2.1. Une fonction $f: I=[a, b] \subset \mathbb{R} \rightarrow \mathbb{C}$ se prolonge en une fonction $\mathscr{C}^{\infty}$ sur $\mathbb{R}$ si et seulement si, pour tout $s>0$, on a

$$
\lim _{k \rightarrow \infty} k^{s} \operatorname{dist}_{I}\left(f, \mathscr{P}_{k}\right)=0 .
$$

La démonstration classique du théorème de Bernstein ne s'applique pas, en général, au cas d'un compact quelconque de $\mathbb{R}^{n}$. En effet, contrairement au cas d'un intervalle de $\mathbb{R}$, il existe un compact $E$ de $\mathbb{R}^{n}$ et une fonction $f: E \rightarrow \mathbb{R}$ de classe $\mathscr{C}^{\infty}$ dans int $E$, tels que $f$ et toutes ses dérivées partielles se prolongent continûment à $E$ et, qu'en même temps, $f$ n'admette aucun prolongement $\mathscr{C}^{\infty}$ sur un voisinage de $E$. En voici un exemple.

Exemple 2.2. Soit $E=E_{1} \cup E_{2} \subset \mathbb{R}^{2}$, où $E_{1}=\left\{(x, y) \in \mathbb{R}^{2}: 0 \leq x \leq 1, g(x) \leq y \leq 1\right\}$ avec $g(x)=\exp (-1 / x)$ si $0<x \leq 1, g(0)=0$, et $E_{2}=[0,1] \times[-1,0]$. Soit

$$
f(x, y)= \begin{cases}g(x) & \text { si }(x, y) \in E_{1} \\ 0 & \text { si }(x, y) \in E_{2}\end{cases}
$$

Alors $f$ n'admet aucun prolongement $\mathscr{C}^{\infty}$ sur un voisinage ouvert de $E$.

Le problème de caractérisation de type Bernstein des fonctions $\mathscr{C}^{\infty}$ en plusieurs variables a été résolu par Pawłucki-Pleśniak ([37]). Dans ce qui suit, on dira que $E$ est déterminant pour les fonctions $\mathscr{C}^{\infty}$ dans $\mathbb{R}^{n}$ si, pour tout $f \in \mathscr{C}^{\infty}\left(\mathbb{R}^{n}\right), f=0$ sur $E$ entraîne $D^{\alpha} f=0$ sur $E$ pour tout $\alpha \in \mathbb{Z}_{+}^{n}$. On montre (Pleśniak [41]) que tout compact 
markovien de $\mathbb{R}^{n}$ est déterminant. Le résultat de Pawłucki-Pleśniak (voir aussi Pleśniak [41]) s'écrit comme suit.

Théorème 2.3. Si un compact $E$ de $\mathbb{R}^{n}$ est déterminant pour les fonctions $\mathscr{C}^{\infty}$, alors les conditions suivantes sont équivalentes :

(i) E est markovien;

(ii) (théorème de type Bernstein) pour toute fonction $f: E \rightarrow \mathbb{R}$, si la suite $\left\{\operatorname{dist}_{E}\left(f, \mathscr{P}_{k}\right)\right\}$ est à décroissance rapide, c'est pour tout $s>0, k^{s} \operatorname{dist}_{E}\left(f, \mathscr{P}_{k}\right) \rightarrow 0$ si $k \rightarrow \infty$, alors $f$ s'étend en une fonction $\mathscr{C}^{\infty}$ dans $\mathbb{R}^{n}$.

Ici $\mathscr{P}_{k}$ désigne l'espace des polynômes de degré $\leq k$ et $\operatorname{dist}_{E}\left(f, \mathscr{P}_{k}\right):=\inf \left\{\|f-p\|_{E}\right.$ : $\left.p \in \mathscr{P}_{k}\right\}$.

L'ensemble $E$ de l'exemple 2.2 est markovien puisqu'il est UPC. Compte tenu du théorème 2.3 , la suite $\left\{\operatorname{dist}_{E}\left(f, \mathscr{P}_{k}\right)\right\}$ n'est pas à décroissance rapide lorsque $f$ est la fonction de cet exemple.

2.2. Applications en analyse différentielle. L'inégalité de Markov permet de construire de façon relativement simple un opérateur linéaire continu d'extension pour les jets de Whitney de classe $C^{\infty}$ (voir Whitney [53]), définis sur un compact markovien de $\mathbb{R}^{n}$. Le problème d'existence d'un tel opérateur a une longue histoire. Les premiers résultats sont dûs à Mityagin [35] (cas d'un intervalle de $\mathbb{R}$ ) et à Seeley [48] (cas d'un demi-espace de $\left.\mathbb{R}^{n}\right)$. Stein [50] a montré qu'un tel opérateur existe si $E$ est l'adhérence d'un domaine borné de $\mathbb{R}^{n}$ dont le bord est localement de classe Lip1. Bierstone [16] a étendu ce résultat au cas où le bord de $E$ est $L i p \alpha$, avec $0<\alpha \leq 1$. Dans [16], Bierstone a également montré qu'un tel opérateur existe quand $E$ est un sous-analytique fermé de $\mathbb{R}^{n}$ tel que int $E \supset$ $E$. Sa méthode a été essentiellement basée sur le célèbre théorème de désingularisation de Hironaka. Une autre méthode, basée sur des résultats de Vogt et Wagner concernant des suites exactes des espaces nucléaires, a été appliquée par Tidten [51] pour montrer l'existence d'un opérateur d'extension pour des fermés de $\mathbb{R}^{n}$ admettant certaines pointes polynomiales. Tous les ensembles mentionnés ci-dessus sont UPC, d'où de Markov. Le théorème suivant de Pawłucki-Pleśniak [38] est une généralisation essentielle des résultats précédents. De plus, il est de caractère constructif.

Théorème 2.4. Soit E un compact markovien de $\mathbb{R}^{n}$. Si, pour tout $k \in \mathbb{N}, L_{k} f$ désigne un polynôme d'interpolation de Lagrange de $f$ de degré $k$, ayant pour nouds des points extrémaux de Fekete-Leja, et $\left(u_{k} ; k \in \mathbb{N}\right)$ désigne une famille appropriée de fonctions de troncature de classe $\mathscr{C}^{\infty}$, alors la formule

$$
L f=u_{1} L_{1} f+\sum_{k=1}^{\infty} u_{k}\left(L_{k+1} f-L_{k} f\right)
$$

définit un opérateur linéaire continu étendant les jets de Whitney de classe $C^{\infty}$ sur $E$ en fonctions de classe $C^{\infty}$ sur l'espace $\mathbb{R}^{n}$ tout entier.

En utilisant des techniques similaires, Zeriahi [54] a montré que si E est un compact de $\mathbb{R}^{n}$ de Markov, alors $\mathscr{C}^{\infty}(E)$ (équipé de la topologie de Whitney) est isomorphe à l'espace 
$\mathscr{S}$ des suites de réels positifs à décroissance rapide, ce qui est une généralisation non triviale du résultat de Mityagin [35] mentionné ci-dessus.

Le théorème de Pawłucki-Pleśniak admet un complément (voir Pleśniak [41]) : la propriété de Markov de $E$ est équivalente à l'existence d'un opérateur d'extension linéaire continu par rapport à la topologie de Jackson de l'espace $\mathscr{C}^{\infty}(E)$. Cette équivalence n'est plus valable lorsque l'espace $\mathscr{C}^{\infty}(E)$ est muni de la topologie de Whitney. En effet, Goncharov [27] a construit un compact non markovien de $\mathbb{R}$ admettant l'existence d'un opérateur d'extension linéaire continu (par rapport à la topologie de Whitney). Pour d'autres résultats concernant l'opérateur d'extension de Pawłucki-Pleśniak voir AltunGoncharov ([1]).

Notons encore que pour qu'un opérateur d'extension linéaire continu existe, il faut quelques restrictions sur les pointes de $E$. En effet, Tidten ([51]) a montré que si $E$ est le compact de l'exemple 1.1, alors il n'existe aucun opérateur d'extension (linéaire continu) de $\mathscr{C}^{\infty}(E)$ dans $\mathscr{C}^{\infty}\left(\mathbb{R}^{2}\right)$.

2.3. Exposant de Markov. Soit $E$ un compact de $\mathbb{R}^{n}$, on pose

$$
\mu(E):=\inf \{r>0: E \text { satisfait à }(\mathrm{M}) \text { avec exposant } r\}
$$

et on l'appelle exposant de Markov de E.

La connaissance de la valeur exacte de $\mu(E)$ permet en particulier de minimiser la perte de régularité dans des problèmes liés à l'extension linéaire des classes de fonctions ultradifférentiables (voir Pleśniak [42], Beaugendre [11]). Si $E$ est un compact connexe de $\mathbb{C}$ contenant au moins deux points différents, alors par Pommerenke $[43], 1 \leq \mu(E) \leq 2$. Si $E$ est un compact de $\mathbb{R}^{n}$ quelconque, en utilisant des propriétés extrémales des polynômes de Tchebyschev, on montre que $\mu(E) \geq 2$. Si $E$ est un compact convexe de $\mathbb{R}^{n}$ d'intérieur non-vide, alors $\mu(E)=2$. Rappellons encore l'exemple de Goetgheluck de $E_{k} \subset \mathbb{R}^{2}$, où $\mu(E)=2 k$. Si $E$ est un compact UPC de $\mathbb{R}^{n}$ à paramètre $m \geq 1$, alors $\mu(E) \leq 2 m$ (voir Baran [3]). Notons finalement que si $E$ est l'ensemble de Zerner (voir exemple 1.1), alors $\mu(E)=\infty$. Il se trouve que l'exposant de Markov est un invariant de «bonnes » applications analytiques. On a (voir Baran-Pleśniak [7])

Théorème 2.5. Soient $E$ un compact de $\mathbb{R}^{n}$ satisfaisant à $(M)$ pour l'exposant $r$ et $f$ une application analytique d'un voisinage $U$ de $E$ dans $\mathbb{R}^{n}$, telle que $f(E)$ soit non pluripolaire (dans $\mathbb{C}^{n}$ ) et, pour tout $x \in E$, $\operatorname{det} d_{x} f \neq 0$. Alors $f(E)$ satisfait à $(M)$ pour le même exposant $r$ que celui de $E$.

2.4. Inégalité de Markov sur les ensembles algébriques de $\mathbb{C}^{n}$. Dans les années récentes, l'inégalité de Markov a été intensivement étudiée sur des sous-variétés algébriques de $\mathbb{R}^{n}$ (travaux de Fefferman-Narasimhan, Roytwarf-Yomdin, Bos-Levenberg-Milman-Taylor, Brudny̌̆, Baran-Pleśniak, Gendre, Kosek, Comte-Yomdin, Erdélyi-Kroó, Kroó-Szabados). Notons ici un résultat important de Sadullaev [46].

Théorème 2.6. Un ensemble analytique $A$ de $\mathbb{C}^{n}$ est algébrique si et seulement si la fonction de Green pluricomplexe $V_{E}^{*}$ est localement bornée sur A pour tout compact non-pluripolaire Ede A. 
Le critère de Sadullaev est crucial pour l'étude des inégalités de type Bernstein-Walsh, Markov ou de van der Corput-Schaake sur les ensembles algébriques (voir Bos-LevenbergTaylor [21], Bos et al. [19, 20], Baran-Pleśniak [8-10]). L'inégalité tangentielle de Markov avec l'exposant égal à 1 caractérise la propriété d'un compact $K$ de $\mathbb{R}^{n}$ d'être morceau d'une variété algébrique. Plus précisement, on a (voir Baran-Pleśniak [9])

Théorème 2.7. Soit $K$ un compact de $\mathbb{R}^{n}$ admettant une paramétrisation analytique $\phi$ : $\mathbb{B}^{m} \rightarrow K$, où $\mathbb{B}^{m}$ est la boule unitée de $\mathbb{R}^{m}(1 \leq m \leq n)$. Alors la dimension de Zariski de $K$ est égale à $m$ si et seulement si on peut trouver une constante $C>0$ telle que, pour tout $x \in K$ et tout polynôme $P \in \mathbb{C}\left[x_{1}, \ldots, x_{n}\right]$,

$$
\left|D_{T(t, v)} P(x)\right| \leq C(\operatorname{deg} P)\|P\|_{K},
$$

où $t \in \phi^{-1}(x) \cap \mathbb{B}^{m}, v \in \mathbb{S}^{m-1}$ et $T(t, v)=D_{v} \phi(t)$ est la dérivée de $\phi$ en direction $v$.

Si $K$ est une sous-variété compacte lisse de $\mathbb{R}^{n}$ de dimension $m, 1 \leq m \leq n$, le théorème ci-dessus a été montré auparavant par Bos et al. [19].

Soient $E$ un compact polynomialement convexe de $\mathbb{C}^{m}$ de type HCP, avec $s$ pour exposant, et $f$ une application analytique non-dégénerée définie sur un voisinage ouvert de $E$, à valeurs dans un sous-ensemble algébrique $\mathbb{M}$ de $\mathbb{C}^{n}$ de dimension $k, 1 \leq k \leq n$. On a alors (Baran-Pleśniak [10])

Théorème 2.8. Il existe une constante $C>0$ telle que, pour tout polynôme $Q \in \mathbb{C}\left[z_{1}, \ldots, z_{n}\right]$ et tout $t \in E$,

$$
\left|D_{T(t, v)} Q(f(t))\right| \leq C_{1} d^{1 / s}\|Q\|_{f(E)} .
$$

Corollaire 2.9. Soit $f$ une application polynomiale de $\mathbb{R}$ dans $\mathbb{R}^{n}$ avec

$$
f^{\prime}(t)=(1-t)^{s_{1}}(1+t)^{s_{2}} Q(t)
$$

où $Q$ est une application polynomiale de $\mathbb{R}$ dans $\mathbb{R}^{n}$ telle que $Q(t) \neq 0$ sur $[-1,1]$. Soit $\alpha=$ $\max \left(s_{1}, s_{2}\right)$. Alors il existe une constante $A>0$ telle que, pour tout polynôme $P \in \mathbb{R}\left[x_{1}, \ldots, x_{n}\right]$ de degré $d$, on ait

$$
\left|D_{T_{x}} P(x)\right| \leq A d^{2+2 \alpha}\|P\|_{N}, \quad \text { pour } x \in N=f([-1,1]),
$$

$\operatorname{avec} D_{T_{x}} P(x)=D_{(Q(t) /\|Q(t)\|)} P(x)$, où $x=f(t)$.

Exemple 2.10. Soient $m$ et $l$ deux entiers positifs premiers entre eux, $m>l \geq 2$. Soit $f(t)=$ $\left(((1+t) / 2)^{l},((1+t) / 2)^{m}\right)$. On a alors

$$
N=f([-1,1])=\left\{(x, y) \in \mathbb{R}^{2}: 0 \leq x, y \leq 1, x^{m}=y^{l}\right\} .
$$


En effet, comme $\alpha=l-1$, le corollaire précédent donne l'inégalité

$$
\left|D_{T_{(x, y)}} P(x, y)\right| \leq A d^{2 l}\|P\|_{N},
$$

pour tout polynôme $P \in \mathbb{R}[x, y]$ de degré $d$.

(Voir aussi Bos et al. [20], Gendre [23].)

Dans [19], on peut trouver un exemple d'une fonction analytique $f:[0,1] \rightarrow \mathbb{R}$ telle que $f([0,1])$ n'admette aucune inégalité de Markov tangentielle dont l'exposant est fini. Ainsi on pourrait penser qu'une telle inégalité n'a lieu que si $f([0,1])$ est morceau d'une variété algébrique. Mais, un contre-exemple a récemment été donné par Bos et al. ([18]).

Théorème 2.11. Soit $T \in \mathbb{R}[x]$ un polynôme quelconque. Pour tout intervalle $[a, b] \subset \mathbb{R}$, on peut trouver une constante $C>0$ telle que, pour tout polynôme $P \in \mathbb{C}[x, y]$, on ait

$$
\max _{x \in[a, b]}\left|\frac{d}{d x} P\left(x, e^{T(x)}\right)\right| \leq C(\operatorname{deg} P)^{4} \max _{x \in[a, b]}\left|P\left(x, e^{T(x)}\right)\right|,
$$

l'exposant 4 étant optimal.

2.5. Remarques finales. La théorie présentée ici témoigne de la force ainsi que de la beauté de l'inégalité classique de Markov. C'est pourquoi je l'appelle « une vénérable dame qui n'a pas perdu de son attrait. 》

Il est difficile de traiter toutes les ramifications possibles de l'inégalité de Markov en plusieurs variables. Pour cette raison, je me suis restreint à sa forme en norme uniforme. Pour les versions $L^{p}$ voir des travaux de Bos-Milman ([22]), Goetgheluck ([25]) et Baran $([4,5])$.

Notons encore que l'inégalité de Markov a été également étudiée en dimension infinie ; nous renvoyons le lecteur aux travaux de Harris ([28]), Sarantopoulos ([47]), MuñozSarantopoulos ([36]), Révész-Sarantopoulos ([45]), Skalyga ([49]) et Baran ([6]).

\section{Bibliographie}

[1] M. Altun and A. Goncharov, A local version of the Pawtucki-Pleśniak extension operator, Journal of Approximation Theory 132 (2005), no. 1, 34-41.

[2] M. S. Baouendi and C. Goulaouic, Approximation of analytic functions on compact sets and Bernstein's inequality, Transactions of the American Mathematical Society 189 (1974), 251-261.

[3] M. Baran, Markov inequality on sets with polynomial parametrization, Annales Polonici Mathematici 60 (1994), no. 1, 69-79.

[4] _ New approach to Markov inequality in $L^{p}$ norms, Approximation Theory, Monogr. Textbooks Pure and Applied Mathematics, vol. 212, Marcel Dekker, New York, 1998, pp. 75-85, (in memory of A. K. Varma).

[5] _ Markov's Inequality in $L^{p}$ norms (I), Jagiellonian University, Cracow, preprint, 2003.

[6] _ Polynomial Inequalities in Banach Spaces (I), Jagiellonian University, Cracow, preprint, 2002.

[7] M. Baran and W. Pleśniak, Markov's exponent of compact sets in $\mathbb{C}^{N}$, Proceedings of the American Mathematical Society 123 (1995), no. 9, 2785-2791.

[8] _ Bernstein and van der Corput-Schaake type inequalities on semialgebraic curves, Studia Mathematica 125 (1997), no. 1, 83-96. 
[9] Characterization of compact subsets of algebraic varieties in terms of Bernstein type inequalities, Studia Mathematica 141 (2000), no. 3, 221-234.

[10] _ Polynomial inequalities on algebraic sets, Studia Mathematica 141 (2000), no. 3, 209219.

[11] P. Beaugendre, Extensions de jets dans des intersections de classes non quasi-analytiques, Annales Polonici Mathematici 76 (2001), no. 3, 213-243.

[12] S. N. Bernstein, Sur l'ordre de la meilleure approximation des fonctions continues par des polynômes de degré donné, Mémoires de l'Académie Royale de Belgique 4 (1912), no. 2, 1-103.

[13] L. Białas and A. Volberg, Markov's property of the Cantor ternary set, Studia Mathematica 104 (1993), no. 3, 259-268.

[14] L. Białas-Cież, Equivalence of Markov's property and Hölder continuity of the Green function for Cantor-type sets, East Journal on Approximations 1 (1995), no. 2, 249-253.

[15] _ Markov sets in $\mathbb{C}$ are not polar, Bulletin of the Polish Academy of Sciences. Mathematics 46 (1998), no. 1, 83-89.

[16] E. Bierstone, Extension of Whitney fields from subanalytic sets, Inventiones Mathematicae 46 (1978), no. 3, 277-300.

[17] E. Bierstone and P. D. Milman, Semianalytic and subanalytic sets, Institut des Hautes Études Scientifiques, Publications Mathématiques 67 (1988), 5-42.

[18] L. P. Bos, A. Brudnyi, N. Levenberg, and V. Totik, Tangential Markov inequalities on transcendental curves, Constructive Approximation 19 (2003), no. 3, 339-354.

[19] L. P. Bos, N. Levenberg, P. D. Milman, and B. A. Taylor, Tangential Markov inequalities characterize algebraic submanifolds of $\mathbb{R}^{n}$, Indiana University Mathematics Journal 44 (1995), no. 1, $115-138$.

[20] _ Tangential Markov inequalities on real algebraic varieties, Indiana University Mathematics Journal 47 (1998), no. 4, 1257-1272.

[21] L. P. Bos, N. Levenberg, and B. A. Taylor, Characterization of smooth, compact algebraic curves in $\mathbb{R}^{2}$, Topics in Complex Analysis (Warsaw, 1992) (P. Jakóbczak and W. Pleśniak, eds.), Banach Center Publ., vol. 31, Polish Academy of Sciences, Warsaw, 1995, pp. 125-134.

[22] L. P. Bos and P. D. Milman, Sobolev-Gagliardo-Nirenberg and Markov type inequalities on subanalytic domains, Geometric and Functional Analysis 5 (1995), no. 6, 853-923.

[23] L. Gendre, Inégalité de Markov tangentielle locale sur les courbes algébriques de $\mathbb{R}^{n}$, Université Paul Sabatier, Toulouse, prépublication, 1998.

[24] P. Goetgheluck, Inégalité de Markov dans les ensembles effilés, Journal of Approximation Theory 30 (1980), no. 2, 149-154.

[25] _ Polynomial inequalities on general subsets of $\mathbb{R}^{N}$, Colloquium Mathematicum 57 (1989), no. $1,127-136$.

[26] P. Goetgheluck and W. Pleśniak, Counter-examples to Markov and Bernstein inequalities, Journal of Approximation Theory 69 (1992), no. 3, 318-325.

[27] A. Goncharov, A compact set without Markov's property but with an extension operator for $\mathfrak{C}^{\infty}$ functions, Studia Mathematica 119 (1996), no. 1, 27-35.

[28] L. A. Harris, A Bernstein-Markov theorem for normed spaces, Journal of Mathematical Analysis and Applications 208 (1997), no. 2, 476-486.

[29] A. Jonsson, Markov's inequality on compact sets, Orthogonal Polynomials and Their Applications (Erice, 1990) (C. Brezinski, L. Gori, and A. Ronveaux, eds.), IMACS Ann. Comput. Appl. Math., vol. 9, Baltzer, Basel, 1991, pp. 309-313.

[30] _ Markov's inequality and zeros of orthogonal polynomials on fractal sets, Journal of Approximation Theory 78 (1994), no. 1, 87-97. 
[31] M. Klimek, Pluripotential Theory, London Mathematical Society Monographs. New Series, vol. 6, The Clarendon Press, Oxford University Press, New York, 1991.

[32] M. Kosek, Hölder continuity property of filled-in Julia sets in $\mathbb{C}^{n}$, Proceedings of the American Mathematical Society 125 (1997), no. 7, 2029-2032.

[33] _ Hölder continuity property of composite Julia sets, Bulletin of the Polish Academy of Sciences. Mathematics 46 (1998), no. 4, 391-399.

[34] S. Łojasiewicz, Ensembles semi-analytiques, Institut des Hautes Études Scientifiques, Bures-surYvette (1964).

[35] B. S. Mityagin, Approximate dimension and bases in nuclear spaces, Russian Mathematical Surveys 16 (1961), no. 4, 59-128.

[36] G. A. Muñoz and Y. Sarantopoulos, Bernstein and Markov-type inequalities for polynomials on real Banach spaces, Mathematical Proceedings of the Cambridge Philosophical Society 133 (2002), no. 3, 515-530.

[37] W. Pawłucki and W. Pleśniak, Markov's inequality and $\mathscr{C}^{\infty}$-functions on sets with polynomial cusps, Mathematische Annalen 275 (1986), no. 3, 467-480.

[38] Extension of $\mathscr{C}^{\infty}$-functions from sets with polynomial cusps, Studia Mathematica 88 (1988), no. 3, 279-287.

[39] R. Pierzchała, UPC condition in polynomially bounded o-minimal structures, Journal of Approximation Theory 132 (2005), no. 1, 25-33.

[40] W. Pleśniak, A Cantor regular set which does not have Markov's property, Annales Polonici Mathematici 51 (1990), 269-274.

[41] _ Markov's inequality and the existence of an extension operator for $\mathscr{C}^{\infty}$-functions, Journal of Approximation Theory 61 (1990), no. 1, 106-117.

[42] _ Extension and polynomial approximation of ultradifferentiable functions in $\mathbb{R}^{n}$, Bulletin de la Société Royale des Sciences de Liège 63 (1994), no. 5, 393-402.

[43] Ch. Pommerenke, On the derivative of a polynomial, The Michigan Mathematical Journal 6 (1959), 373-375.

[44] Q. I. Rahman and G. Schmeisser, Analytic Theory of Polynomials, London Mathematical Society Monographs. New Series, vol. 26, The Clarendon Press, Oxford University Press, Oxford, 2002.

[45] S. G. Révész and Y. Sarantopoulos, On Markov constants of homogeneous polynomials over real normed spaces, East Journal on Approximations 9 (2003), no. 3, 277-304.

[46] A. Sadullaev, An estimate for polynomials on analytic sets, Mathematics of the USSR - Izvestiya 20 (1983), no. 3, 493-502.

[47] Y. Sarantopoulos, Bounds on the derivatives of polynomials on Banach spaces, Mathematical Proceedings of the Cambridge Philosophical Society 110 (1991), no. 2, 307-312.

[48] R. T. Seeley, Extension of $\mathfrak{C}^{\infty}$ functions defined in a half space, Proceedings of the American Mathematical Society 15 (1964), 625-626.

[49] V. I. Skalyga, Analogues of Markov's inequality in normed spaces, Mathematical Notes 75 (2004), no. 5-6, 739-743.

[50] E. M. Stein, Singular Integrals and Differentiability Properties of Functions, Princeton Mathematical Series, no. 30, Princeton University Press, New Jersey, 1970.

[51] M. Tidten, Fortsetzungen von $\mathscr{C}^{\infty}$-Funktionen, welche auf einer abgeschlossenen Menge in $\mathbb{R}^{n}$ definiert sind, Manuscripta Mathematica 27 (1979), no. 3, 291-312.

[52] V. Totik, Markoff constants for Cantor sets, Acta Scientiarum Mathematicarum (Szeged) 60 (1995), no. 3-4, 715-734.

[53] $\mathrm{H}$. Whitney, Analytic extensions of differentiable functions defined in closed sets, Transactions of the American Mathematical Society 36 (1934), no. 1, 63-89. 


\section{Inégalité de Markov en plusieurs variables}

[54] A. Zeriahi, Inegalités de Markov et développement en série de polynômes orthogonaux des fonctions $\mathscr{C}^{\infty}$ et $\mathscr{A}^{\infty}$, Several Complex Variables (Stockholm, 1987/1988) (J. F. Fornaess, ed.), Math. Notes, vol. 38, Princeton University Press, New Jersey, 1993, pp. 683-701.

[55] M. Zerner, Développement en séries de polynômes orthonormaux des fonctions indéfiniment différentiables, Comptes Rendus de l'Académie des Sciences. Série I. Mathématique 268 (1969), 218-220.

Wiesław Pleśniak : Institut de mathématiques, Université Jagellonne de Cracovie, UL. Reymonta 4, 30-059 Kraków, Pologne

Courrier électronique : wieslaw.plesniak@im.uj.edu.pl 


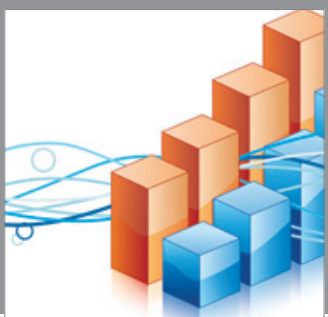

Advances in

Operations Research

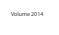

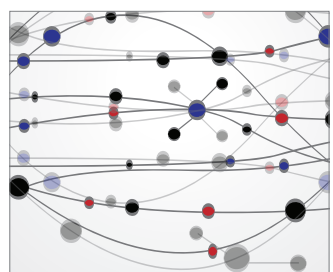

\section{The Scientific} World Journal
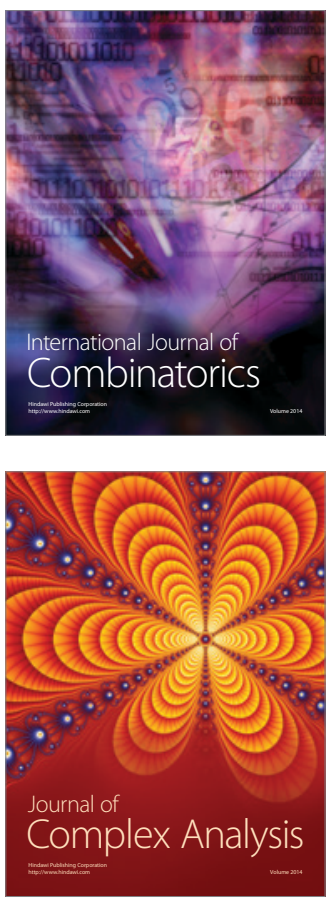

International Journal of

Mathematics and

Mathematical

Sciences
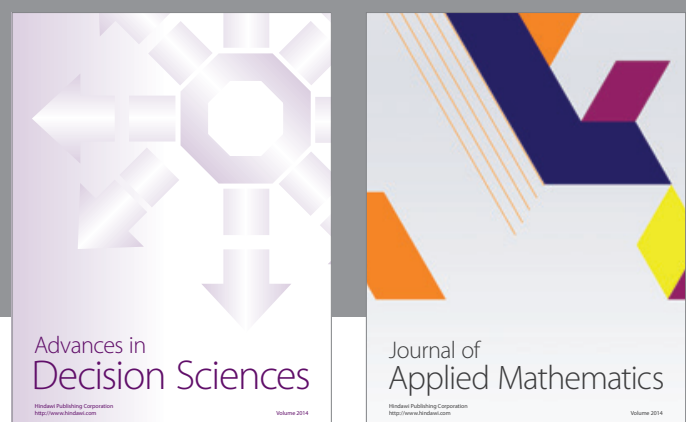

Journal of

Applied Mathematics
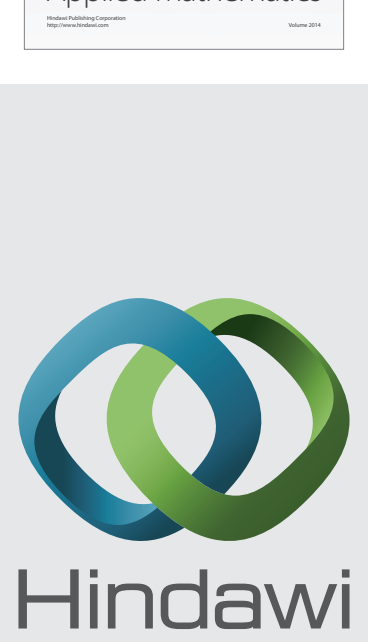

Submit your manuscripts at http://www.hindawi.com
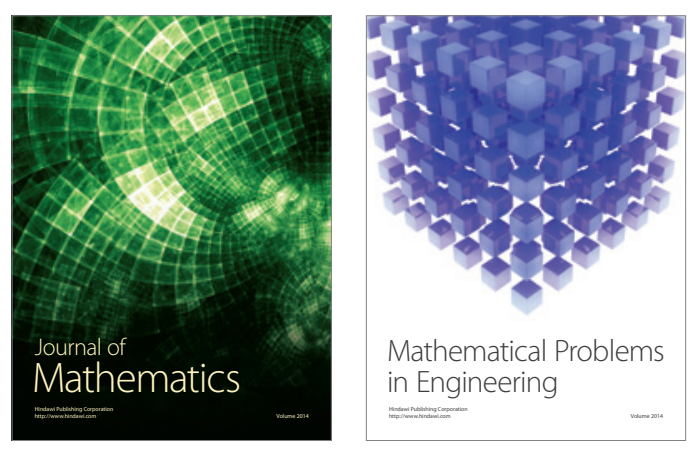

Mathematical Problems in Engineering
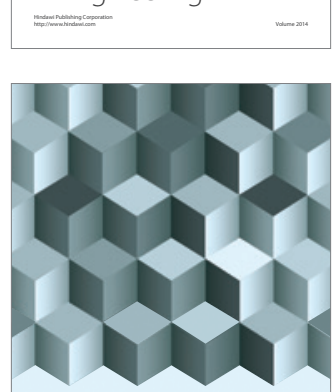

Journal of

Function Spaces
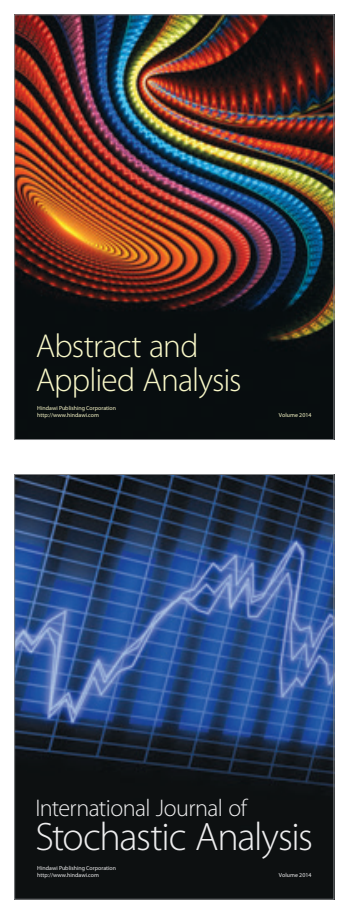

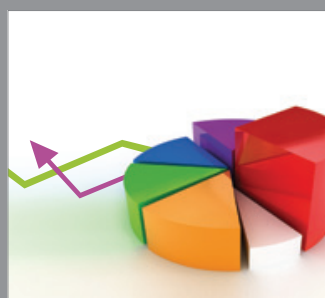

ournal of

Probability and Statistics

Promensencen
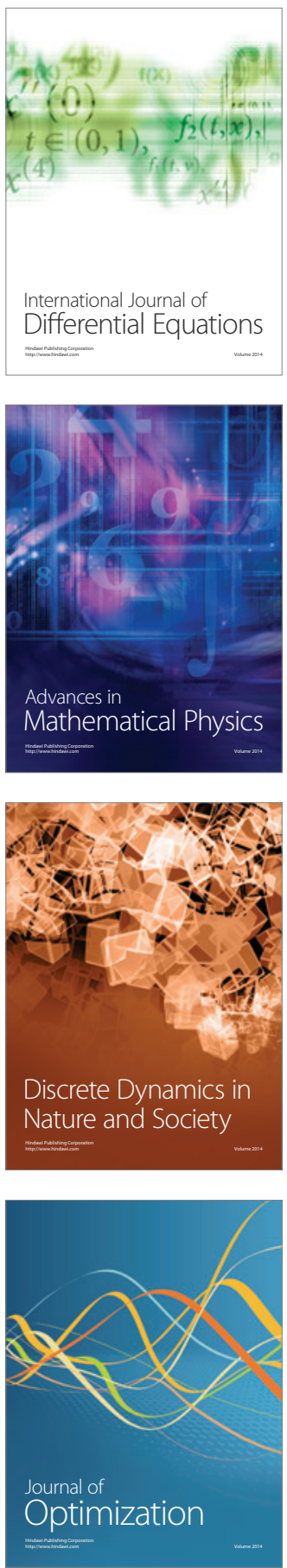